\title{
The Response of Work Function of Thin Metal Films to Interaction with Hydrogen
}

\author{
R. Duś*, E. Nowicka and R. Nowakowski \\ Institute of Physical Chemistry, Polish Academy of Sciences \\ Kasprzaka 44/52, 01-224 Warszawa, Poland
}

The aim of this paper is to summarize the results of experiments carried out at our laboratory on the response of the work function of several thin films of transition metals and rare earth metals to interaction with molecular hydrogen. The main focus concerns the description of surface phenomena accompanying the reaction of hydride formation as a result of the adsorbate's incorporation into the bulk of the thin films. Work function changes $\Delta \Phi$ caused by adsorption and reaction concern the surface, hence this experimental method is appropriate for solving the aforementioned problem. A differentiation is made between the work function changes $\Delta \Phi$ due to creation of specific adsorption states characteristic of hydrides, and $\Delta \Phi$ arising as a result of surface defects and protrusions induced in the course of the reaction. The topography of thin metal films and thin hydride films with defects and protrusions was illustrated by means of atomic force microscopy. For comparison, the paper discusses work function changes caused by $\mathrm{H}_{2}$ interaction with thin films of metals which do not form hydrides (for example platinum), or when this interaction is performed under conditions excluding hydride formation for thermodynamic reasons. Almost complete diminishing of $\Delta \Phi$ was observed, in spite of significant hydrogen uptake on some rare earth metals, caused by formation of the ordered $\mathrm{H}-\mathrm{Y}-\mathrm{H}$ surface phase.

PACS numbers: 68.43.- $\mathrm{h}, 82.30 . \mathrm{Rs}, 73.30 .+\mathrm{y}$

\section{Introduction}

It is commonly known that at temperatures above $10 \mathrm{~K}$ molecular hydrogen dissociates on the surface of transition metals. Under appropriate conditions hydrogen interaction with transition or rare earth metals leads to new compounds $\mathrm{MeH}_{x}$ hydrides. Metal hydrides are nonstoichiometric compounds with hydrogen distributed along the interstitial sites of the metal, held in place by a combination

*corresponding author; e-mail: dus@ichf.edu.pl 
of covalent and ionic bonding [1]. Metal hydrides are created when equilibrium hydrogen pressure $P_{\text {eq }}$ characteristic of $\mathrm{MeH}_{x}$ is exceeded. $P_{\text {eq }}$ differs significantly for various transition metals [1], for example reaching $35 \mathrm{MPa}$ at room temperature for rhodium hydride [2], and $1.1 \mathrm{kPa}$ for palladium hydride [1]. The equilibrium pressure strongly depends on temperature. For example, for palladium hydride it drops to $10^{-2} \mathrm{~Pa}$ at $78 \mathrm{~K}[3]$. The decrease in $P_{\text {eq }}$ with decreasing temperature makes it possible to study surface phenomena during transition metal hydride formation using UHV apparatus, thus ensuring a clean metal surface.

Hydrogen is an element which has the ability to lose or accept an electron, thus becoming a proton or forming a negative ion of the stable configuration of a helium atom. Experimental results collected in the literature show that both states of hydrogen can be present on the surface and also in the bulk of various metals. $\mathrm{H}^{-}$anions are present without any doubt in hydrides of alkaline metals [4], whereas it has been experimentally proved that in the bulk of hydrides of several transition metals e.g. palladium, niobium or vanadium, hydrogen is clearly protonic [5]. On the other hand, there is experimental evidence that negatively charged hydrogen species arise in the bulk of trihydrides of some rare earth metals [6]. The creation of positively charged atomic hydrogen adspecies on the surface of transition metals was predicted theoretically long ago by Grimley [7]. The validity of this theoretical prediction was confirmed experimentally some years ago [8-12].

The mechanism of incorporation of the hydrogen adsorbate below the surface is not well understood, particularly in the case when hydrogen anions are present in the bulk, as is the case in rare earth metal trihydrides. The mobility of protons in metals is much greater than that of negative hydrogen ions. Hence the question arises whether, in the course of hydride formation, the negatively charged hydrogen species arise directly on the surface of the adsorbents and slowly incorporate into the bulk, or positively charged hydrogen adatoms are first created on the outer surface, and quickly penetrate below it, reaching the interstitial sites within the subsurface region. Then, the rearrangement of metal electrons occurs, leading to the creation of negatively charged hydrogen species. Each of these two mechanisms should be accompanied by a different change of the electric charge distribution on the surface.

Adsorption-induced work function changes of metals $\Delta \Phi$ is a physical property which is strongly affected by the electric charge distribution on the very surface. Thus, precise measurements of $\Delta \Phi$ caused by hydrogen adsorption could help to answer the above question. However, the electric charge distribution on the surface can also be influenced by the surface topography. One example is the case when adsorption followed by a chemical reaction results in a new phase with changes of the surface structure. When the lattice constant of the new phase differs from that characteristic of the original metal, this can be a source of stress. The stress, by changing the volume of the surface region, can affect the electron density within it, hence influencing the electric charge distribution. Moreover, in 
the case of thin films this change of volume also influences the metal's adhesion to the support. As a consequence, protrusions or defects can be created on the previously flat surface. These features influence the electric charge distribution and can even locally change the polarization of the adsorbate [12]. Thus, to answer the question on the response of work function changes to the interaction of hydrogen with metals, $\Delta \Phi$ measurements should be accompanied by determination of the surface mesostructure. The use of scanning tunneling microscopy (STM) or atomic field microscopy (AFM) is very helpful here. Application of thin films of various metals deposited on selected substrates allowing for the preparation of a variety of surface topographies can result in a set of interesting information.

We have found that in many cases thin metal films deposited under UHV conditions, thus having a clean surface, are reactive toward hydride formation, while single crystals are highly resistive. For this reason further studies were carried out using thin metal films.

\section{Experimental}

To study the surface phenomena which occur during hydrogen interaction with transition or rare earth metal surfaces under conditions when hydrides could be formed, an experimental method capable of distinguishing between different hydrogen adspecies over wide pressure and temperature intervals is required. A measuring circuit with a short response time which allows the observation of the surface process dynamics is useful. We have found that these requirements are fulfilled during simultaneous measurement of work function changes $\Delta \Phi$ and $\mathrm{H}_{2}$ pressure $P$. Describing adsorption, it is convenient to use the notation of surface potential (SP) instead of work function changes $\Delta \Phi$. By definition $\mathrm{SP}=-\Delta \Phi$. Hence an increase in SP corresponds to positively charged adspecies arising on the surface. Precise SP measurements allow to determine the electrostatic features of the adsorbate and elucidate the dynamics of elementary surface processes that occur during adsorption or reaction. When the processes are carried out at a constant, calibrated volume, simultaneous $\mathrm{H}_{2}$ pressure recording allows to correlate these features with gas uptake $\left(N_{\mathrm{H}}\right)$ determined volumetrically. Thin films were prepared by complete evaporation of metals of known weight from a tungsten heater. The system used was a UHV glass system capable of routinely reaching $(1-2) \times 10^{-8} \mathrm{~Pa}$ during thin film deposition and allowing to work at $\mathrm{H}_{2}$ pressure of up to $\approx 10 \mathrm{~Pa}$.

The surface potential response for hydrogen adsorption or isothermal desorption caused by lowering the $\mathrm{H}_{2}$ pressure was studied by means of the static capacitor method. The principles of this method and the construction of the cell have been described previously $[13,14]$. SP was monitored continuously using a fully automatic, sensitive $(1 \mathrm{mV})$, short response time $(1 \mathrm{~ms})$, high stability $(1 \mathrm{mV} / \mathrm{h})$ electronic circuit [15]. Spectroscopically pure hydrogen, additionally purified by diffusion through a palladium thimble, was used. $\mathrm{H}_{2}$ was introduced 
in successive calibrated doses into the static capacitor maintained at a chosen, constant temperature and disconnected from pumps. The hydrogen pressure in the static capacitor in the range from $10^{-4}$ to $10 \mathrm{~Pa}$ was monitored by means of an ultrasensitive, short response time Pirani-type gauge immersed in a liquid nitrogen bath. A typical $\mathrm{H}_{2}$ dose introduced into the static capacitor and not adsorbed would increase the pressure up to $10^{-1} \mathrm{~Pa}$. Thus, the consumption of hydrogen following every successive $i$-th dose could be precisely determined. Knowing the weight of the thin films and $\mathrm{H}_{2}$ uptake, the number ratio of hydrogen atoms to metal atoms $\left(N_{\mathrm{H}} / N_{\mathrm{Me}}\right)$, altogether on the surface and in the bulk, can be determined at every step of the process and correlated with SP. Examination of the time-dependent features of $\mathrm{SP}_{i}(t)$ and $P_{i}(t)$ associated with the $i$-th introduced dose allows to study the kinetics of surface processes at a determined $\left(N_{\mathrm{H}} / N_{\mathrm{Me}}\right)$ ratio. Equilibrium was achieved after the $i$-th introduced dose when $\mathrm{d}\left(\mathrm{SP}_{i}\right) / \mathrm{d} t=0$ and $\mathrm{d} P_{i} / \mathrm{d} t=0$. Having the equilibrium surface potential $\mathrm{SP}_{i, \text { eq }}$ and equilibrium $\mathrm{H}_{2}$ pressure $P_{i \text {,eq }}$ for every hydrogen dose, two important relations can be obtained at a chosen, constant temperature: (1) the surface potential isotherm $\mathrm{SP}_{\text {eq }}=$ $\sum_{i} \mathrm{SP}_{i, \text { eq }}=f_{1}\left(N_{\mathrm{H}} / N_{\mathrm{Me}}\right)$ and (2) the thermodynamic isotherm $P_{\mathrm{eq}}=\sum_{i} P_{i, \mathrm{eq}}=$ $f_{2}\left(N_{\mathrm{H}} / N_{\mathrm{Me}}\right)$. On the basis of these two functions, distinct forms of hydrogen adsorbate can be determined at every step of the process.

For AFM studies, the thin metal films (palladium or platinum) were deposited on a flat microscope glass (Menzel Glaser) under the same conditions as described above. The samples were covered with oxygen to protect them against adsorption of any impurities from the ambient atmosphere during their transportation from the UHV apparatus into the AFM system (TopoMetrix, TMX 2000). A closed AFM cell was used, enabling controlled flow of several gases $\left(\mathrm{H}_{2}, \mathrm{O}_{2}\right.$, Ar) over the samples under atmospheric pressure. It is commonly known that due to the catalytic reaction oxygen can be replaced with hydrogen on the surface of $\mathrm{Pt}$ and Pd [16]. Thus, the protective oxygen adsorbate can be removed from the sample surface and the influence of hydrogen on the surface topography can be studied.

\section{Experimental results and discussion}

\subsection{Work function (surface potential) response to hydrogen adsorption on thin metal films in the absence of hydrides}

It is well known that in spite of the highest available $\mathrm{H}_{2}$ pressure (50 MPa) being applied in studies of hydrogen interaction with platinum, the hydride $\mathrm{PtH}_{x}$ has not been obtained. On the other hand, dissociative adsorption of hydrogen on a platinum surface occurs easily [16]. Mignolet found that hydrogen adsorption on thin Pt film deposited on a support maintained at low temperature caused a decrease in surface potential at low coverage $\Theta$ followed by an increase in SP accompanying the increase in the adsorbate population [17]. 


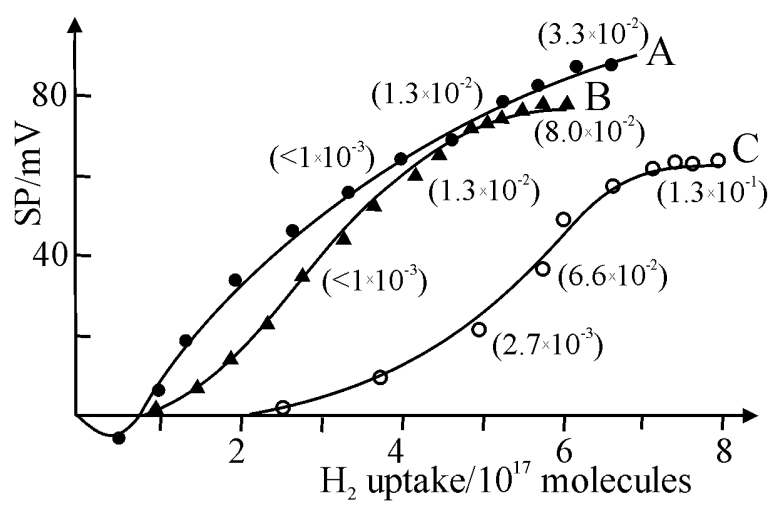

Fig. 1. Surface potential isotherms $\mathrm{SP}=f\left(\mathrm{H}_{2}\right.$ uptake $)$ for hydrogen adsorption on thin platinum film deposited under UHV conditions on glass maintained at $273 \mathrm{~K}$. Isotherms marked A, B, and C were obtained at 78, 195, and $273 \mathrm{~K}$, respectively. Equilibrium $\mathrm{H}_{2}$ pressure corresponding to several hydrogen uptakes is expressed in $\mathrm{Pa}$ [18].

We performed systematic studies of hydrogen adsorption on thin Pt films deposited under UHV conditions on a glass support maintained at $78 \mathrm{~K}$ and slightly sintered (at $300 \mathrm{~K}$ ), and also on thin Pt films deposited on the support kept at $273 \mathrm{~K}[18,19]$. The surface potential isotherm for hydrogen adsorption on thin Pt film condensed at $273 \mathrm{~K}$ is shown in Fig. 1. Contrary to Mignolet's findings, a very slight decrease in SP was observed at low population of the adsorbate and it was followed by a significant increase in surface potential accompanying the increase in coverage. This behavior of SP for the $\mathrm{H}_{2}$-thin $\mathrm{Pt}$ film system is similar to that known for adsorption on low index planes of platinum single crystals [20]. Examination of the surface potential isotherm $\mathrm{SP}=f\left(\mathrm{H}_{2}\right.$ uptake $)$ showed that the positively polarized hydrogen deposited on the thin $\mathrm{Pt}$ film is not uniform. Under equilibrium pressure below $2 \times 10^{-2} \mathrm{~Pa}$ it obeys Langmuir's isotherm for dissociative adsorption, while under higher pressure the isotherm corresponding to molecular hydrogen adsorption is valid. On the basis of these two isotherms the adsorption heats were estimated to be $33 \mathrm{~kJ} / \mathrm{mol}$ for the atomic positively polarized species and $4 \mathrm{~kJ} / \mathrm{mol}$ for the molecular adorbate [18].

The films condensed at low temperature should contain a much higher density of surface defects. This expectation was confirmed some years later by AFM data. Figure 2 shows the image of thin Pt film condensed at $78 \mathrm{~K}$ and sintered at room temperature. The image was obtained under an Ar atmosphere after treatment of the sample with hydrogen in the cell of the microscope. The high population of surface defects is clearly seen in the cross-section presented in the inset. One can notice that majority of surface corrugation associated with defects on thin Pt film achieved a few nanometers. Defects of this dimension are known to be active in catalytic reactions. The defects are resistive against interaction with hydrogen and oxygen. The surface structure of the Pt film observed on the 


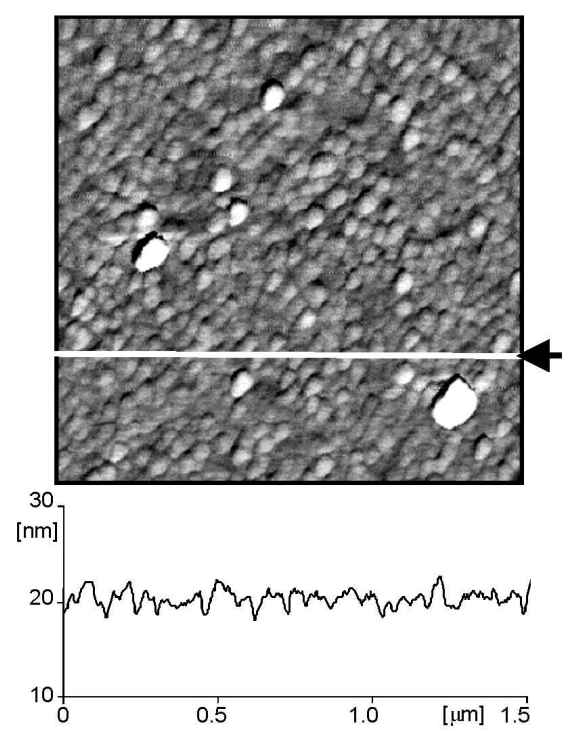

Fig. 2. AFM image of thin Pt film deposited on glass at $78 \mathrm{~K}$ and sintered at $298 \mathrm{~K}$.

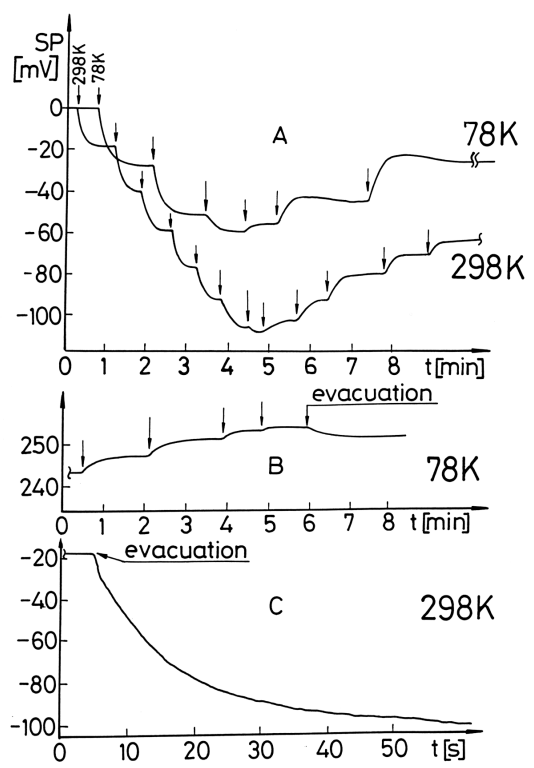

Fig. 3. Course of SP changes during hydrogen adsorption at $78 \mathrm{~K}$ and $298 \mathrm{~K}$ on thin $\mathrm{Pt}$ film deposited at $78 \mathrm{~K}$ on glass under UHV conditions. Arrows indicate successive $\mathrm{H}_{2}$ doses introduction into the static capacitor. For better illustration of the course of adsorption the figure is divided into sections (A), (B), and (C) [19]. 
image did not exhibit any change as a result of interaction with hydrogen flowing through the cell under pressure $\approx 100 \mathrm{kPa}$. The course of surface potential changes in the process of hydrogen adsorption on thin $\mathrm{Pt}$ film deposited at $78 \mathrm{~K}$ is shown in Fig. 3. For better clearness only $\mathrm{SP}(t)$ features which were registered as a result of successive hydrogen dose introduction into the static capacitor maintained at two temperatures: 78 and $298 \mathrm{~K}$ are shown. However, $\mathrm{H}_{2}$ interaction with thin Pt films at several other temperatures was also examined. At the beginning of adsorption a significant decrease in SP was registered, while successive doses caused an increase in surface potential. This was very similar to the result obtained by Mignolet [17]. We ascribe this phenomenon to the high density of surface defects on thin Pt film condensed at low temperature.

The possibility of inverse polarization for hydrogen adatoms placed around surface defects on a platinum surface was theoretically discussed by Anzelm [21]. It is important to notice that each successive dose of hydrogen caused a fast change of surface potential to a stable value, accompanied by a constant pressure of the gas phase. No SP transients were observed. This means that both positively and negatively polarized hydrogen adspecies are stable on a thin Pt film surface and do not penetrate into the bulk. It is not well understood yet why positively charged hydrogen adspecies do not incorporate below the platinum surface as they do in the case of many other transition metals in the process of hydride formation. One can notice in Fig. 3 that isothermal evacuation of the static capacitor at $298 \mathrm{~K}$ caused complete desorption of the positively polarized hydrogen adspecies, while it did not alter the population of the negatively polarized ones. This is a direct evidence that surface defects increase the binding energy of hydrogen adatoms to the platinum surface. The real area of the thin Pt film surface can be exactly determined on the basis of the reciprocal hydrogen-oxygen titration [16, 22]. Thus, knowing hydrogen uptake the dependence of SP on coverage $\Theta$ on platinum film can be described. This dependence is shown in Fig. 4 for several temperatures. The population of surface defects indicated by the uptake of negatively polarized hydrogen adspecies (the negative pole of the dipole pointing away from surface) is apparently higher when detected during adsorption carried out at higher temperature. One should remember, however, that surface potential is an additive function, thus the registered SP is a superposition of the signal due to negatively polarized, strongly bounded hydrogen adatoms present, as we suggest, on the sites around the surface defects, and also due to the signal from weakly bound, positively polarized adspecies located on the non-defected sites. At higher temperatures, under the same hydrogen pressure, the population of weakly bounded adspecies is diminished in comparison with low temperature adsorption, thus the registered SP is caused mainly by negatively polarized adspecies present at the defects, thus is more negative. Thin Pt film condensed at higher temperature is expected to have a much smaller density of defects than that deposited at low temperature. Hence our suggestion concerning the influence of surface defects and 


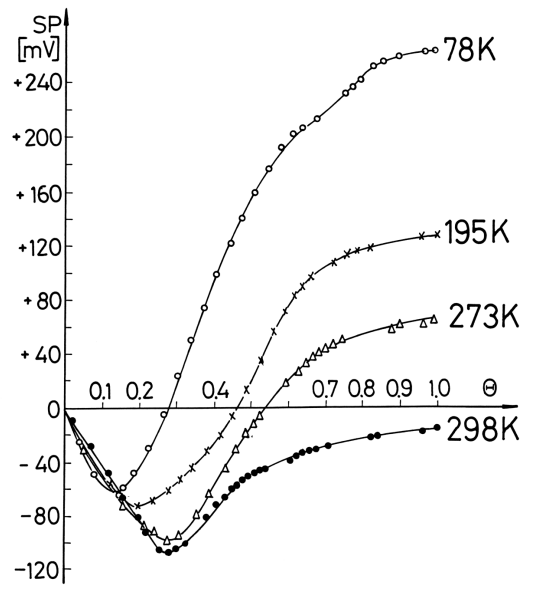

Fig. 4. Surface potential isotherms $\mathrm{SP}=f(\Theta)$ for hydrogen adsorption at several temperatures on thin Pt film deposited on glass at $78 \mathrm{~K}$ [19].

the related alteration of platinum surface atoms' electronic structure on the sign of hydrogen adatom polarization seems to be rational.

Almost parallel to our investigations of thin Pt films Christmann and Ertl studied hydrogen adsorption at low temperature $(130 \mathrm{~K})$ on highly packed faces of platinum single crystals (111) and (110) and on the plane $\mathrm{Pt}(997)$ characterized by the presence of terraces between atomic steps [20]. They observed a monotonic increase in SP accompanying the increase in coverage $\Theta$ on $\operatorname{Pt}(111)$ and $\operatorname{Pt}(100)$, while $\mathrm{H}$ deposit on a stepped $\mathrm{Pt}(997)$ surface resulted in a decrease in SP at low population followed by its decrease with the increase in $\Theta$. The authors ascribed the difference in the sign of polarization of hydrogen adatoms compared with adsorption on $\mathrm{Pt}(111)$ to the altered electronic properties of the $\mathrm{Pt}$ atoms at the edge of the steps. It should be expected that other defects of dimension around a few nanometers present on a flat surface of platinum could act similarly as the edges of terraces on stepped surfaces, locally changing the binding energy and even the sign of the electric polarization of the adsorbate. Hence dependence of the surface potential on coverage for hydrogen deposit on condensed thin Pt film should exhibit a similar feature as that found for the stepped surface of a platinum single crystal. The decrease in SP could be however much more pronounced, since the density of surface defects is much higher. As described above, this phenomenon was actually observed.

It has been well established that dissociative adsorption of hydrogen on the majority of transition metal surfaces, carried out under thermodynamic conditions which preclude hydride formation, occurs with the decrease in surface potential [16]. The platinum case described above is one of few exceptions. Figure 5 shows the course of surface potential changes registered as a result of hydrogen adsorption performed at $298 \mathrm{~K}$ on thin palladium film [3]. 


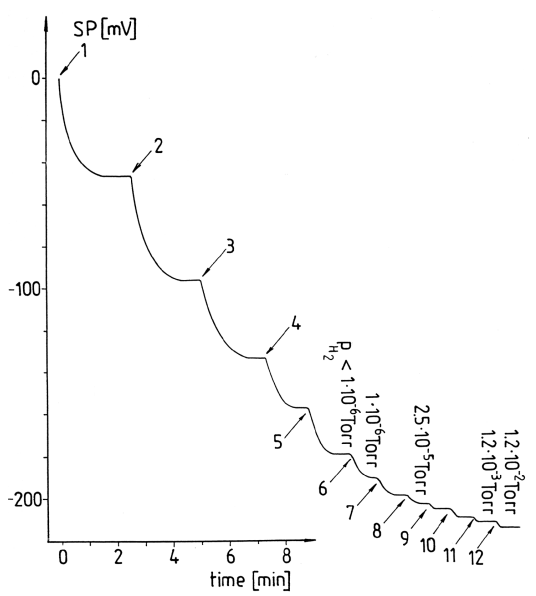

Fig. 5. Course of SP changes during hydrogen adsorption at $298 \mathrm{~K}$ on thin Pd film deposited on glass at $78 \mathrm{~K}$ and sintered at $350 \mathrm{~K}$. Arrows indicate successive hydrogen doses introduction into the static capacitor. Equilibrium $\mathrm{H}_{2}$ pressure corresponding to several doses is marked [19].

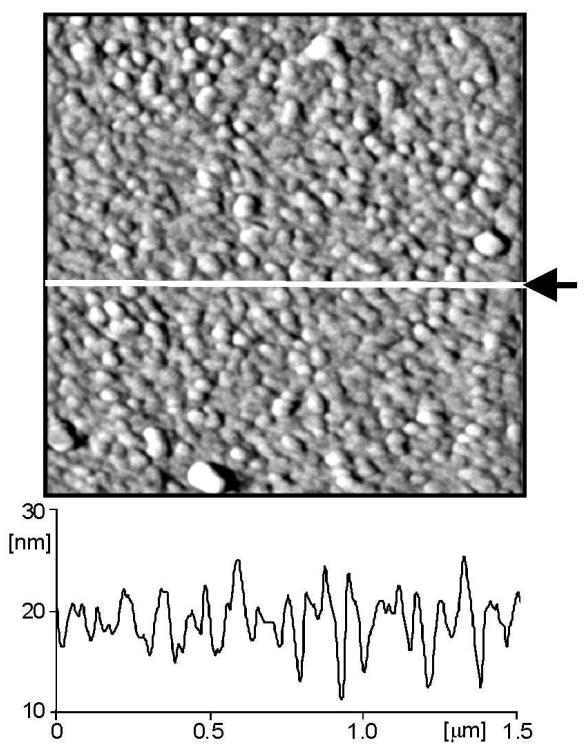

Fig. 6. AFM image of thin Pd film deposited at $78 \mathrm{~K}$ on glass under UHV conditions and sintered at $350 \mathrm{~K}$.

The AFM image of the thin Pd film is demonstrated in Fig. 6. There is a similarity between the topography of the thin $\mathrm{Pt}$ and $\mathrm{Pd}$ films. However the profiles are different. On the surface of thin Pd films defects predominate of much higher dimension than those observed on platinum. $\mathrm{H}_{2}$ was intro- 
duced in successive calibrated doses into the static capacitor disconnected from pumps, with simultaneous measurement of the surface potential SP and gas phase pressure $P_{\mathrm{H}_{2}}$. A fast decrease in SP followed by a stable value of surface potential was observed as a result of every successive dose's admission. The equilibrium value of $P_{\mathrm{H}_{2}}$ corresponding to some arbitrarily chosen doses is marked in the graph. The adsorption was finished when $\Delta \mathrm{SP}_{i}$ caused by the $i$-th dose approached zero. Corresponding pressure reached 1.6 Pa. This is almost three orders of magnitude below the equilibrium pressure required for palladium hydride formation at $298 \mathrm{~K}$. Thus, under the described experimental conditions we are dealing exclusively with hydrogen adsorption on a thin Pd film surface. The area of the thin Pd film can be determined on the basis of hydrogen-oxygen reciprocal titration [23]. The coverage corresponding to every $i$-th $\mathrm{H}_{2}$ dose and $\Delta \mathrm{SP}_{i}$ can thus be determined. Hence the surface potential isotherm $\Delta \mathrm{SP}(\Theta)$ can be constructed. A monotonic decrease in surface potential with increasing coverage is observed. A monotonic course of the surface potential isotherm occurs when only one state of the adsorbate is present on the surface. This requirement is fulfilled under experimental conditions during the adsorption described above, carried out at $298 \mathrm{~K}$. Other adsorption states of hydrogen on thin $\mathrm{Pd}$ film arise when adsorption is performed at a lower temperature [24]. Additional adsorption states of hydrogen are usually induced by an increase in coverage and are manifested as kinks in the surface potential isotherm. One good example is hydrogen adsorption on a thin iron film [14] as presented

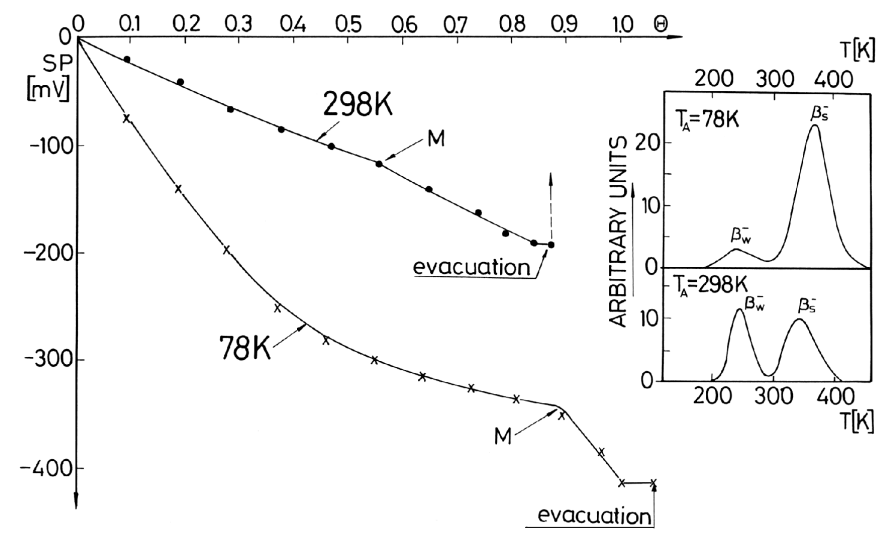

Fig. 7. Surface potential isotherms for hydrogen adsorption at $78 \mathrm{~K}$ and $298 \mathrm{~K}$ on thin Fe film deposited on glass at $78 \mathrm{~K}$ and sintered at $400 \mathrm{~K}$. The coverage is calculated taking maximal hydrogen uptake at $78 \mathrm{~K}$ as a monolayer. Point denoted $\mathrm{M}$ corresponds to the arising of the induced $\beta_{\mathrm{w}}^{-}$state of the adsorbate. Increase in SP caused by isothermal desorption of the weakly bounded $\beta_{\mathrm{w}}^{-}$adspecies at $298 \mathrm{~K}$ is shown, as well as the lack of SP changes during evacuation at $78 \mathrm{~K}$ corresponding to stability of the adsorbate at $78 \mathrm{~K}$. Thermal desorption spectrum indicating two states of the adsorbate is shown in the inset [19]. 
in Fig. 7. A decrease in SP was registered during creation of an adsorbate. At low coverage, a strongly adsorbed state arose, referred to as $\beta_{\mathrm{s}}^{-}$. The increase in hydrogen uptake induced a new, weakly bound state of the adsorbate referred to as $\beta_{\mathrm{w}}^{-}$. The dipole moment values of these two states differ, leading to a kink in the $\operatorname{SP}(\Theta)$ graph. The thermal desorption spectrum shown in the inset confirms the existence of two states of hydrogen on the thin iron film.

\subsection{Work function (surface potential) response to hydride creation within thin films of transition metals}

The creation of transition metal hydrides is usually accompanied by a change of the crystallographic structure of the original metal [1] or at least by a change of its lattice constant. This results in the appearance of stress. In some cases (e.g. $\mathrm{VH}_{x}, \mathrm{NbH}_{y}$ ) the stress is so high that a bulk metal is transferred into a fine powder. This effect can be partially avoided when thin films are applied, due to the relaxation caused by adhesion to the support. The extent of the relaxation influences the topography of thin films and hence also the electron density distribution on the surface.

Palladium hydride exhibits the same crystallographic structure as the original metal, however the lattice constant increases by $3.5 \%$ [25]. It was found that within a thin palladium film deposited on a glass support under UHV conditions, atomic concentration of hydrogen as high as $\mathrm{H} / \mathrm{Pd} \approx 0.98$ can be easily reached at $78 \mathrm{~K}$ under pressure as low as $\approx 10^{-1} \mathrm{~Pa}$. According to the phase diagram for the $\mathrm{Pd}-\mathrm{H}_{2}$ system, this concentration corresponds to the pure $\beta$-phase of palladium hydride [25].

Figure 8 shows the course of SP changes in the process of $\mathrm{PdH}_{x}(0.01<$ $x<0.98$ ) formation at $78 \mathrm{~K}[3]$. For better illustration the presentation is divided into three sections corresponding to the characteristic steps of the reaction. At the beginning of the process several $\mathrm{H}_{2}$ doses introduced into the static capacitor caused a decrease in SP when equilibrium conditions between the gas phase and the adsorbate were reached (Fig. 8a). This corresponds to the formation of negatively polarized adspecies. These adspecies are referred to as $\beta^{-}$to emphasize their negative polarization. Figure 8 shows that apart from the first dose, the SP response to the admission of subsequent doses was manifested by a transient of the positive SP. This must be associated with the fast arising and slower vanishing of positively polarized adspecies. These positively polarized adspecies are referred to as $\beta^{+}$. The features of this graph are a good fit for Grimley's theoretical prediction mentioned above [7]. According to his model the increase in the hydrogen adsorbate population is accompanied by an increase in the splitting between evenand odd-induced electron states. There is a critical population above which the lower state merges into the metal conduction band. Thereafter, the donation of electrons from hydrogen adatoms into unoccupied states in the conduction band occurs, leading to the formation of positively charged hydrogen adsorbate. The nature of hydrogen adspecies arising in this way on the surface is similar to that 


\section{Graph I}

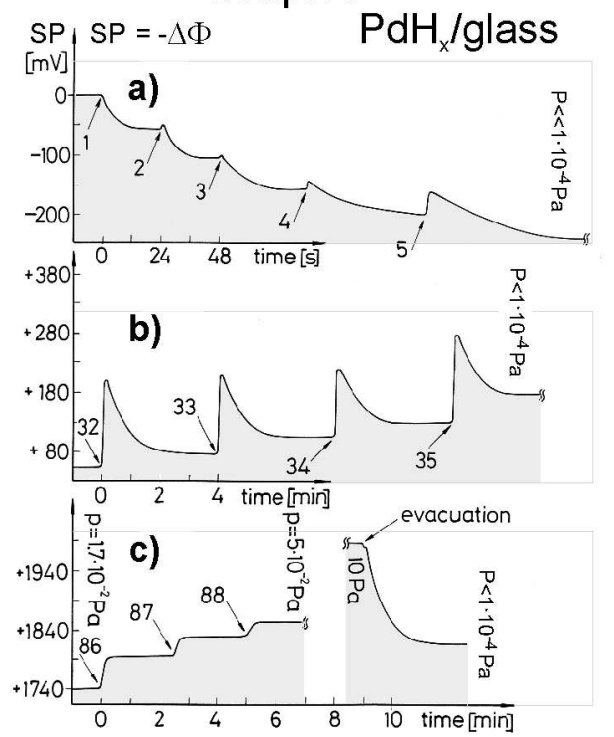

Graph II

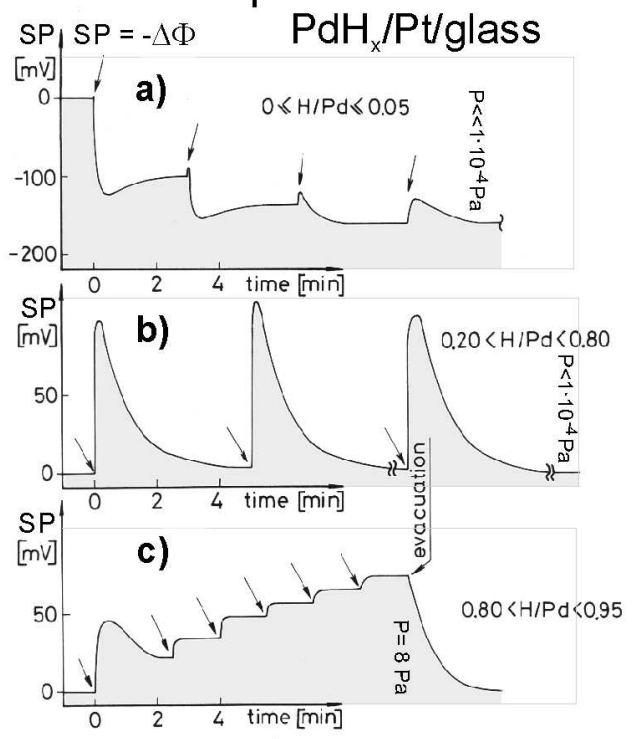

Fig. 8. Course of SP changes in the process of palladium hydride formation at $78 \mathrm{~K}$ within thin Pd film deposited under UHV conditions at $78 \mathrm{~K}$ on glass (graph I), and within thin $\mathrm{Pd}$ film deposited on a support consisted in thick Pt film deposited on glass (graph II). Figures are divided into sections (a), (b), and (c) to better illustrate distinguished steps of the process [12].

present in the bulk. Thus, due to the concentration gradient the $\beta^{+}$adspecies incorporate quickly below the surface. This is observed as the positive SP transient. Analysis of SP transients shows that the rate of surface potential decrease fits the diffusion equation very well. This allows the determination of the diffusion coefficient for the $\beta^{+}$adspecies incorporation below the surface. It was found that its value only slightly depends on the $\mathrm{H} / \mathrm{Pd}$ ratio in the bulk and reaches $(2-4) \times 10^{-13} \mathrm{~cm}^{2} / \mathrm{s}$. This property suggests a tunneling mechanism for the incorporation. Such a conclusion was later supported by studies of palladium deuteride formation [3]. Starting from $\mathrm{H} / \mathrm{Pd}$ concentration of $\approx 0.08$, the SP transients were not complete. The SP equilibrium value was increasingly more positive with every $\mathrm{H}_{2}$ dose introduced (Fig. 8b). Many $(\approx 80)$ successive $\mathrm{H}_{2}$ doses were consumed with the characteristic SP transients until atomic concentration $\mathrm{H} / \mathrm{Pd}$ reached 0.7. The corresponding equilibrium $\mathrm{SP}$ was as high as $+1700 \mathrm{mV}$. The phase diagram for the hydrogen-palladium system shows that at $78 \mathrm{~K}$ this is the boundary of the region for coexistence of two phases: $\alpha$ (disordered solution of hydrogen in $\mathrm{Pd}$ ) and $\beta$ (ordered $\mathrm{PdH}$ ). In spite of heavy uptake of hydrogen the equilibrium pressure was of the order of $1 \times 10^{-4} \mathrm{~Pa}$. Further hydrogen doses, however, caused a different effect in SP changes, mainly a monotonic increase in SP (Fig. 8c) with 
every successive dose. Also the equilibrium pressure increased now with every $\mathrm{H}_{2}$ dose. At hydrogen equilibrium pressure of around $10^{-1} \mathrm{~Pa}$ the $\mathrm{H} / \mathrm{Pd}$ concentration reached $\approx 0.98$, accompanied by $\Delta \mathrm{SP}=+1990 \mathrm{mV}$. Further $\mathrm{H}_{2}$ doses did not result in any measurable change of SP. Isothermal evacuation decreased the surface potential by $\approx 150 \mathrm{mV} . \mathrm{H}_{2}$ redosing rebuilt the adsorbate exactly. This procedure can be repeated many times with a very good reproducibility. The very high increase in SP in the process of $\mathrm{PdH}_{x}(0.1<x<0.98)$ was really surprising. It could correspond to the presence of highly protonized hydrogen adspecies on the surface, or it could be caused by a drastic change of thin Pd film topography due to stress generation in the course of hydride formation. In the second case the change of the support for thin Pd film should influence cohesion and topography. As mentioned earlier, high cohesion would increase relaxation of the stress generated in the reaction of hydride formation and consequently diminish thin film deformation. We performed an experiment with deposition of thin $\mathrm{Pd}$ film on a support which consisted of thick platinum film pre-deposited "in situ" under UHV conditions on glass at $78 \mathrm{~K}$ and sintered at $373 \mathrm{~K}$. High cohesion between thin films of platinum and palladium is very well known. Further details of the experimental procedure were the same as described earlier. The course of SP changes is presented in Fig. 8 (graph II). Complete SP transients were registered now, while all other features of the process were the same as for Pd deposited on glass. However this difference is very significant.

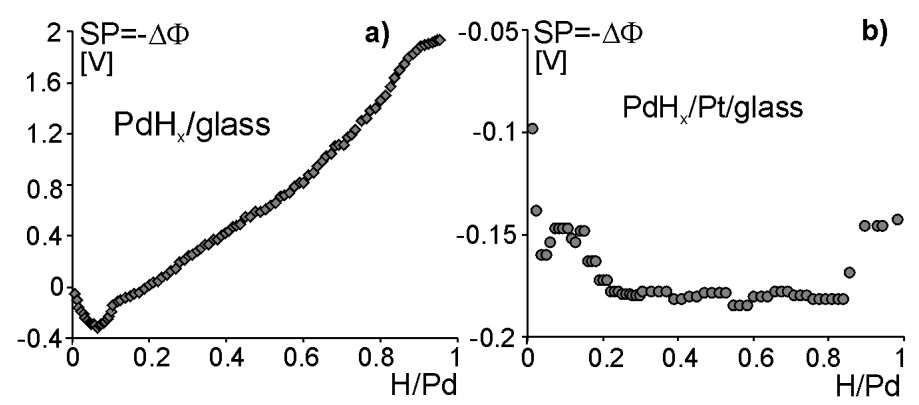

Fig. 9. SP isotherms for palladium hydride formation at $78 \mathrm{~K}$ within thin $\mathrm{Pd}$ films deposited on glass (a) and within thin Pd film deposited on thick Pt film on glass (b) $[12]$.

Figure 9 shows the dependence of $\mathrm{SP}$ on the $\mathrm{H} / \mathrm{Pd}$ ratio registered in the course of the above-described two experiments. In the case of hydride formation in thin $\mathrm{Pd}$ film deposited on platinum, a plateau of $\mathrm{SP}$ was registered within $\mathrm{H} / \mathrm{Pd}$ atomic concentration of $0.2-0.8$, while in the case of $\mathrm{PdH}_{x}$ created in $\mathrm{Pd}$ deposited on glass, a strong increase in SP was observed. This suggested the role of surface defects generated in the reaction of hydride formation. To gain a better understanding of this phenomenon the AFM experiment was performed with a 
thin Pd film deposited on microscopic glass by means of a mask half-covered with a thin Pt film. Thus, we had two different adsorbents in one sample. Next the sample was transported into the AFM cell and hydrogen flow at $298 \mathrm{~K}$ under $\mathrm{H}_{2}$ pressure $\approx 100.1 \mathrm{kPa}$ was performed.

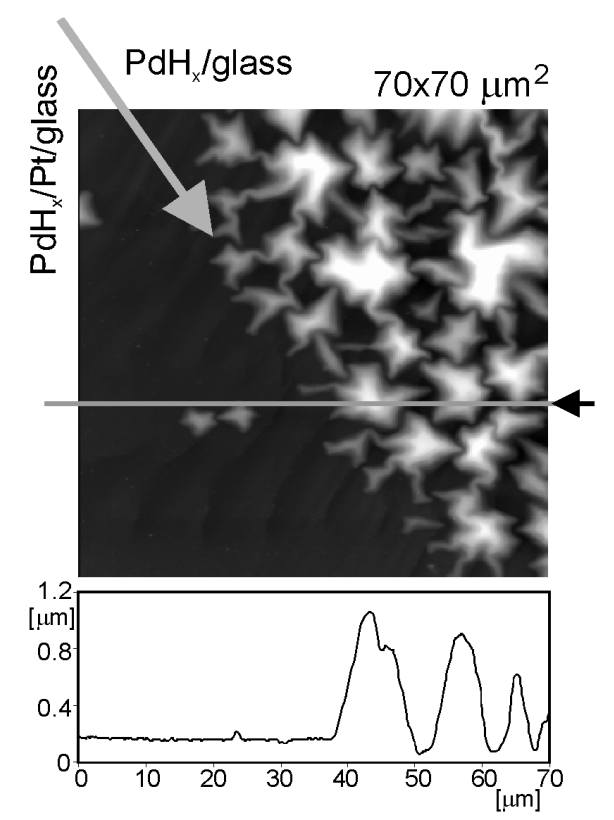

Fig. 10. AFM image and profile of $\mathrm{PdH}_{x}$ formed at $298 \mathrm{~K}$ within thin $\mathrm{Pd}$ film deposited on glass and on the support consisted of thick Pt film deposited on glass. Protrusions formed within the $\mathrm{PdH}_{x} /$ glass are clearly seen as well as their absence in the $\mathrm{PdH}_{x} / \mathrm{Pt} /$ glass system [12].

The result is presented in Fig. 10. Within the thin Pd film deposited on glass, distinct protrusions were generated due to $\mathrm{PdH}$ formation, while the film deposited on platinum remained smooth. Protrusions generated due to the stress arising in the reaction of palladium hydride formation could be completely removed when the hydride was decomposed due to lowering of $\mathrm{H}_{2}$ pressure below the equilibrium pressure over $\mathrm{PdH}$ at $298 \mathrm{~K}$ by changing the composition of the gas phase in the AFM cell for argon. Thus a strong increase in SP in the process of $\mathrm{PdH}$ formation should definitely be attributed to surface defects, not to the special adsorption state of hydrogen. Surface potential features like positive SP transients, and particularly the presence of a positively polarized weakly bounded adsorbate, seems to be common for the creation of other transition metal hydrides at low temperature (around $78 \mathrm{~K}$ ). These features were observed for hydrides of titanium, vanadium, and niobium $[8,10,11]$. In the case of these metals, however, hydride formation changes the crystallographic structure and this process is 
activated, thus the amount of hydrogen consumed at low temperature is diminished to $\mathrm{H} / \mathrm{Me} \approx 0.2-0.4$. The nature of the above-mentioned reversibly adsorbed hydrogen deposit on the hydride surface is particularly interesting. The question arises whether this is a unique state of weakly bounded atomic hydrogen found only on the surface of hydrides or a molecular adsorbate which could exist in the second layer accompanying a high concentration of hydrogen on the surface. A reversibly adsorbed deposit can be obtained at $78 \mathrm{~K}$ within the pressure interval 0.1-1 Pa, thus with our sensitive Pirani-type gauge we can determine the surface potential isotherm and thermodynamic isotherm for this selected state: $\mathrm{SP}_{\text {eq }}\left(n_{\mathrm{a}}\right)$ and $n_{\mathrm{a}}\left(P_{\text {eq }}\right)$ where $n_{\mathrm{a}}$ corresponds to the population of the reversibly adsorbed adspecies. We can also determine the kinetics of adsorption of the reversibly adsorbed deposit by simultaneously measuring $\mathrm{SP}(t)$ and $P_{\mathrm{H}_{2}}(t)$ during a calibrated constant flow of hydrogen into the static capacitor containing palladium hydride with previously removed weakly bounded adspecies. It was found that the reversibly adsorbed hydrogen adspecies on palladium hydride and on the hydrides of other transition metals ( $\mathrm{Ti}, \mathrm{Nb}, \mathrm{V}, \mathrm{Ni}$ ) are strongly polarized. The dipole moments for these systems calculated according to MacDonald and Barlow's equation [26] for mobile interacting dipoles reached high values of 0.21-0.35 D [27]. An analysis showed that the isothermal adsorption and desorption rates of the reversibly adsorbed hydrogen on transition metal hydrides at $78 \mathrm{~K}$ do not fit any simple kinetic equation. It should be expected, however, that in the case of weak adsorption any interaction within the adsorbate could be important. Thus the activation energies for adsorption $E_{\mathrm{a}}$ and desorption $E_{\mathrm{d}}$ should be corrected by the introduction of a term associated with electrostatic interaction. The correction term is equal to the electrical work $\left\{b\left[\mathrm{SP}\left(n_{\mathrm{a}}\right)\right]\right\}$ required to charge the adsorbate up to the measured surface potential $\mathrm{SP}\left(n_{\mathrm{a}}\right)$ dependent on the adsorbent population $n_{\mathrm{a}}$, while $b$ is a proportionality factor characteristic of the adsorbate-adsorbent system. The coverage-dependent electrostatic energy should accelerate desorption and inhibit adsorption. Thus

$$
E_{\mathrm{d}}=E_{\mathrm{d}(0)}-b\left[\operatorname{SP}\left(n_{\mathrm{a}}\right)\right] \quad \text { and } \quad E_{\mathrm{a}}=E_{\mathrm{a}(0)}+b\left[\operatorname{SP}\left(n_{\mathrm{a}}\right)\right],
$$

where $E_{\mathrm{d}(0)}$ and $E_{\mathrm{a}(0)}$ are the activation energies at very low coverage.

We found that the second order kinetic equation for adsorption and desorption with the activation energies corrected according to Eq. (1) fit the experimental data well. This suggests dissociative adsorption and associative desorption, thus the presence of a weakly bounded atomic hydrogen adspecies at $78 \mathrm{~K}$ on the outer surface of the above-mentioned hydrides. This suggestion is confirmed independently under equilibrium conditions on the basis of the $\mathrm{SP}_{\text {eq }}\left(n_{\mathrm{a}}\right)$ and $n_{\mathrm{a}}\left(P_{\text {eq }}\right)$ isotherms determined for the selected hydrogen deposit reversibly adsorbed at $78 \mathrm{~K}$. Under equilibrium conditions the rates of dissociative adsorption and associative desorption are equal, thus 


$$
\begin{gathered}
Z S_{0}\left(1-n_{\mathrm{a}} / n_{\mathrm{a}, \max }\right)^{2} \exp \left(-\left(E_{\mathrm{a} 0}+b \mathrm{SP}\left(n_{\mathrm{a}}\right)\right) / k T\right) P_{\mathrm{eq}} \\
=k_{\mathrm{d}}\left(n_{\mathrm{a}} / n_{\mathrm{a}, \max }\right)^{2} \exp \left(-\left(E_{\mathrm{d} 0}-b \operatorname{SP}\left(n_{\mathrm{a}}\right)\right] / k T\right),
\end{gathered}
$$

where $Z$ is the collision factor, $S_{0}$ is the initial sticking probability and $n_{\mathrm{a}, \max }$ is the maximal population of the reversibly adsorbed species on the hydride surface calculated on the basis of the surface potential isotherm.

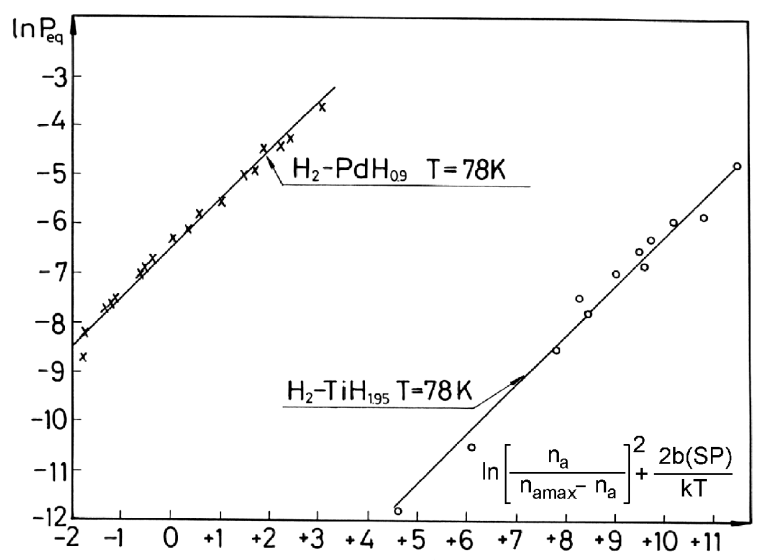

Fig. 11. Examination of $P_{\text {eq }}$ dependence on $n_{\mathrm{a}}$ and SP for weakly bound hydrogen deposit present at $78 \mathrm{~K}$ on $\mathrm{PdH}_{0.9}$ and $\mathrm{TiH}_{1.95}$ according to Eq. (2).

Accordingly, a plot of $\ln \left(P_{\text {eq }}\right)$ versus $\left\{2 \ln \left(n_{\mathrm{a}} /\left(n_{\mathrm{a}, \max }-n_{\mathrm{a}}\right)\right]+2 b \mathrm{SP}\left(n_{\mathrm{a}}\right) / k T\right\}$ should yield a straight line with a slope of unity. This requirement was actually observed for all investigated transition metals forming hydrides. As an example, Fig. 11 shows the result of examination of Eq. (2) for reversibly adsorbed hydrogen adspecies on the surfaces of $\mathrm{PdH}_{0.9}$ and $\mathrm{TiH}_{1.95}$ at $78 \mathrm{~K}$.

\subsection{Work function (surface potential) response to hydride creation in thin films of rare earth metals}

It has been established that hydrogen interaction at low temperature with some rare earth metals and also with yttrium leads to the creation of an ordered $\alpha^{\prime}$ phase consisting of symmetrical $\mathrm{Y}-\mathrm{H}-\mathrm{Y}$ species with the axis oriented perpendicularly to the surface [28]. This phase, stable at low temperature, inhibits the creation of hydrogen-rich hydrides $\mathrm{YH}_{3}$ in the bulk. It was interesting to examine the surface potential response for such a phenomenon. Thin yttrium films were deposited on glass under UHV conditions at $78 \mathrm{~K}$. The area of the thin film was determined by means of the Brunauer-Emmett-Teller (BET) method in the course of a blind experiment. $\mathrm{H}_{2}$ was introduced in successive calibrated doses into the static capacitor maintained at $78 \mathrm{~K}$, with simultaneous measurement of SP and pressure as described above. The admission of several hydrogen doses resulted in adsorption determined volumetrically, while no change of SP was noticed [29], as 


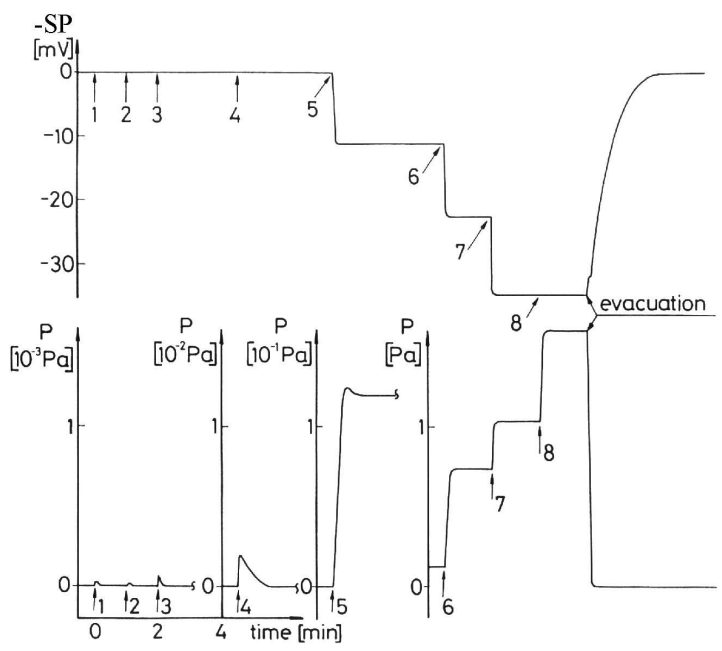

Fig. 12. The signals of SP changes and $\mathrm{H}_{2}$ pressure caused by successive hydrogen dose introduction into the static capacitor maintained at $78 \mathrm{~K}$ containing thin yttrium film. The isothermal desorption of weakly adsorbed adspecies detected by SP changes is clearly seen [29].

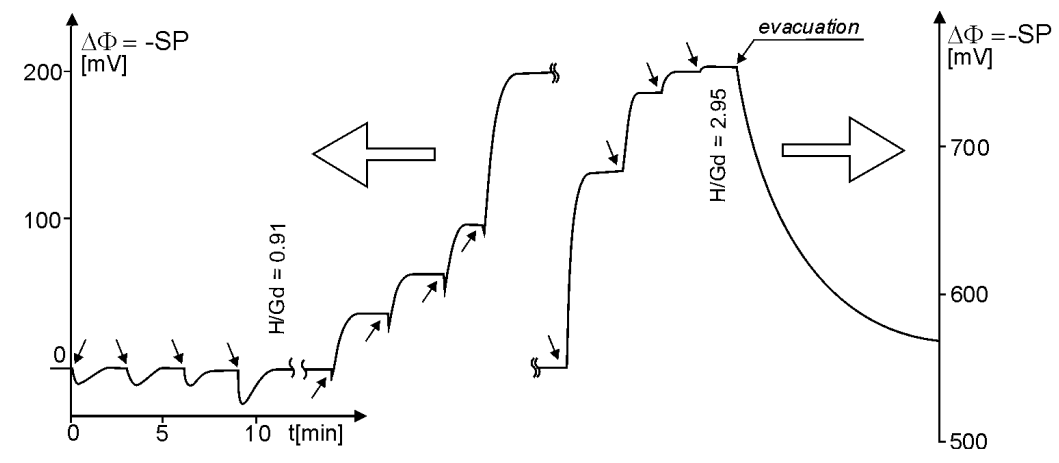

Fig. 13. Course of $\mathrm{SP}$ in the process of $\mathrm{GdH}_{x}$ formation at $298 \mathrm{~K}$. Arrows indicate introduction of successive $\mathrm{H}_{2}$ doses into the static capacitor containing thin Gd film. Diminishing of the positive SP transients and the change of the sign of the polarization of the adsorbate induced by an increase in hydrogen uptake can be noticed [30].

shown in Fig. 12. This must correspond to the creation of non-polar short-range order units $\mathrm{H}-\mathrm{Y}-\mathrm{H}$ with axis perpendicular to the surface as suggested in the literature [28]. Further doses of hydrogen caused a rapid pressure increase accompanied by a small increase in SP. This part of the hydrogen deposit was reversibly adsorbed and could be removed by lowering the pressure. The kinetics of desorption fits the first order kinetic equation very well. This suggests the presence of molecular hydrogen adspecies [29]. At higher temperatures the $\alpha^{\prime}$ phase is not sta- 
ble. Studying hydrogen interaction with thin Gd film at $298 \mathrm{~K}$ [29] we observed, as shown in Fig. 13, that successive $\mathrm{H}_{2}$ doses resulted in small positive SP transients, arising due to positively polarized hydrogen adatoms creation on the outer surface and their quick penetration into the bulk. With increasing hydrogen uptake, at atomic concentration $\mathrm{H} / \mathrm{Gd}>0.91$, each successive dose caused a fast increase in SP followed by its slow decrease until a constant value of SP was reached. A large amount of hydrogen was consumed with this feature of SP changes, until the $\mathrm{H} / \mathrm{Gd}$ atomic ratio reached 2.1. Further adsorption up to $\mathrm{H} / \mathrm{Gd} \approx 2.95$ was registered as a monotonic decrease in SP. A similar effect was observed at $298 \mathrm{~K}$ for yttrium. This is the evidence that in the process of rare earth metal hydride formation, negatively polarized hydrogen adspecies can be created directly on the surface with increasing coverage, probably forming a precursor state for trihydride generation in the bulk.

\section{Conclusions}

Measurements of work function changes (or surface potential) accompanied by pressure registration allowing correlation with hydrogen uptake can bring a lot of information concerning the states of the adsorbate arising at the selected steps of $\mathrm{H}_{2}$ interaction with thin metal films. However, determination of the response of surface topography on this interaction is required for realistic interpretation of the observed phenomena.

Particularly interesting is arising at low temperature $(78 \mathrm{~K})$ on surfaces of some transition metal hydrides (e.g. $\mathrm{PdH}_{0.9}, \mathrm{TiH}_{1.85}, \mathrm{VH}_{1.8}$ ) a weakly bound, positively polarized state of atomic hydrogen. On the other hand, at this temperature on the surfaces of rare earth metals, hydrogen forms an ordered layer of $\mathrm{H}-\mathrm{Me}-\mathrm{H}$ units of very small dipole moment, thus manifested by very small surface potential. On the surfaces of rear earth metal trihydrides, a weakly bounded, negatively polarized form of atomic hydrogen exists.

\section{References}

[1] Y. Fukai, The Metal-Hydrogen System, Springer-Verlag, Berlin 1993.

[2] M. Tkacz, J. Chem. Phys. 108, 2084 (1998).

[3] R. Duś, E. Nowicka, Z. Wolfram, Surf. Sci. 216, 1 (1989).

[4] B. Baranowski, Physica B 265, 16 (1999).

[5] R.C. Brouwer, R. Griessen, Phys. Rev. Lett. 62, 1760 (1989).

[6] F.J.A. den Broeder, S.J. van der Molen, M. Kremers, J.N. Huibertus, D.G. Nagengast, A.T. van Gogh, W.H. Huisman, N.J. Koeman, B. Dam, J.H. Rector, S. Plota, M. Haaksama, R.M. Jungblut, P.A. Duine, R. Grissen, Nature 394, 656 (1998).

[7] T.B. Grimley, in: Chemisorption, Ed. W.E. Garner, Butterworth, London 1957, p. 17.

[8] R. Duś, E. Nowicka, Langmuir 12, 1520 (1996). 
[9] E. Nowicka, R. Duś, Langmuir 12, 1520 (1997).

[10] R. Duś, E. Nowicka, Z. Wolfram, Langmuir 14, 5487 (1998).

[11] R. Duś, Surf. Sci. 52, 440 (1975).

[12] R. Duś, R. Nowakowski, E. Nowicka, J. Alloys Comp. 404/406, 284 (2005).

[13] T. Delchar, A. Eberhagen, F.C. Tompkins, J. Sci. Instrum. 40, 105 (1963).

[14] E. Nowicka, R. Duś, Surf. Sci. 144, 665 (1984).

[15] A. Bachtin, Vacuum 12, 519 (1985).

[16] G.C. Bond, Catalysis by Metals, Academic Press, London 1962.

[17] J.C.P. Mignolet, J. Chim. Phys. 17, 333 (1957).

[18] R. Duś, F.C. Tompkins, J. Chem. Soc. Faraday Trans. I 71, 930 (1975).

[19] R. Duś, E. Nowicka, Prog. Surf. Sci. 67, 139 (2001).

[20] K. Christmann, G. Ertl, Surf. Sci. 60, 365 (1976).

[21] J. Anzelm, Surf. Sci. 108, 561 (1981).

[22] G. Wedler, Chemisorption: an Experimental Approach, Butterworths, London 1976.

[23] R. Duś, W. Lisowski, Surf. Sci. 59, 141 (1976).

[24] E. Nowicka, Z. Wolfram, R. Duś, Surf. Sci. 247, 248 (1991).

[25] H. Friske, E. Wicke, Ber. Bunsenges. Phys. Chem. 77, 48 (1973).

[26] J. Ross MacDonald, C.A. Barlow, J. Chem. Phys. 39, 412 (1963).

[27] E. Nowicka, R. Duś, J. Alloys Comp. 253-254, 506 (1997).

[28] P. Vajda, in: Handbook of the Physics and Chemistry of Rare Earth, Vol. 20, Ch. 137, Eds. K.A. Geseider, L. Eiring, Elsevier, Amsterdam 1995, p. 220.

[29] R. Duś, E. Nowicka, Langmuir 16, 584 (2000).

[30] R. Nowakowski, E. Nowicka, R. Duś, Appl. Surf. Sci. 254, 4346 (2008). 